\title{
Ultrasound and electromyography \& electrical stimulation-guided botulinum toxin injection of salivary glands for drooling: A case report with a description of the technique
}

\author{
Esra Giray @ Evrim Karadağ-Sayg1@, İlker Yağcı@ \\ Department of Physical Medicine and Rehabilitation, Marmara University School of Medicine, Istanbul, Turkey
}

Received: August 19, 2019 Accepted: September 30, 2019 Published online: March 04, 2021

A 34-year-old female who had traumatic brain injury caused by a gunshot 13 year ago was seen for anterior drooling. Previously, she underwent several blind botulinum toxin injections into the parotid and submandibular gland which did not work sufficiently. Drooling severity grade was $5 / 5$, drooling frequency grade was $4 / 4$ as assessed by the Drooling Severity and Frequency Scale (DSFS) and she complained about bad odor and soaking of clothes and using hand towels daily. Ultrasound and electromyography \& electrical stimulation-guided 25 IU of onabotulinum toxin A injection (a total of $50 \mathrm{IU}$ ) was applied to each parotid gland at two different injection points using a 25 -gauge $50 \times 0.5-\mathrm{mm}$ needle electrode using the in-plane technique. Both written and oral informed consents of the patient were obtained before the injection. Ultrasound was used to differentiate the parotid parenchyma having a brighter and homogenous echogenicity (resembling thyroid gland echogenicity) due to its high fatty content. In addition, color or power mode of ultrasound was used to assess the vascular anatomy of the parotid and injection side carefully. When the needle was placed in the accurate position, the absence of motor unit potential was used to confirm that the needle was placed into the parotid gland and electrical stimulation was done to check the proximity of the main branches of the facial nerve to visualize whether there was a twitch in the mimetic muscles. Once no electromyographic signals and contraction of muscles were seen, injection was applied safely and accurately (Figure 1). The patient reported remarkable improvements after botulinum toxin injections. Drooling severity improved to $3 / 5$ and severity improved to $2 / 4$ on DFSS, and the number of soaked towels decreased to only one.

Drooling is common among several neurological disorders such as cerebral palsy, Parkinson's disease,
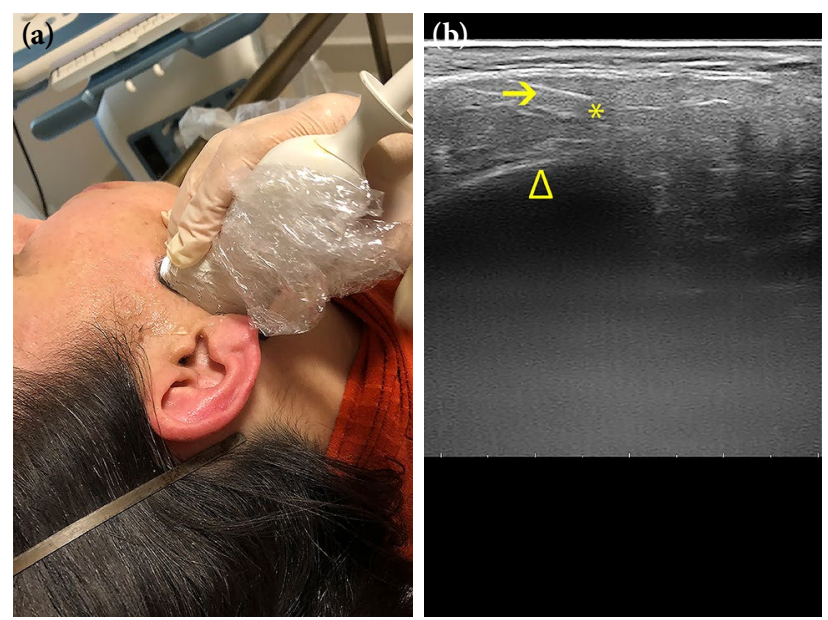

Figure 1. The illustration depicts probe positioning and botulinum toxin injection into parotid gland. (a). The position of the probe (b) Needle (arrow), mandibular ramus (arrowhead) and parotid parenchyma (asterisk).

Corresponding author: Esra Giray, MD. Marmara Üniversitesi Pendik Eğitim ve Araştırma Hastanesi, Fiziksel Tip ve Rehabilitasyon Kliniği, 34899 Pendik, İstanbul, Türkiye. e-mail: esra.giray@marmara.edu.tr 
and amyotrophic lateral sclerosis, which are the main diseases which physiatrists are one of the leading physicians involved in the management. ${ }^{[1]}$ Drooling can be seen either as anterior drooling, unintentional leaving of saliva from mouth to outside of the body, or posterior drooling, the invisible spill of saliva from mouth through pharyngeal isthmus and, then, to respiratory or digestive tract, inside the body. ${ }^{[2]}$ Anterior drooling causes psycho-social impairment, skin problems, infections, bad odor, dehydration, dentation problems, and wet clothes and tools, while posterior drooling may result in morbidity by posing a risk for aspiration pneumonia. ${ }^{[1,2]}$

Botulinum toxin injection of the salivary glands parotid and submandibular glands is one of the medical treatments in the management of drooling. It is applied by neurologists, ear, nose and throat specialists, physiatrists, and interventional radiologists using blind and ultrasound-guided techniques. It has been demonstrated that ultrasound-guided injections are more accurate and effective. ${ }^{[3,4]}$ Both parotid and submandibular glands are found in the neighborhood of important anatomical structures which may be related to complications. The facial artery crosses the submandibular gland parenchyma, while the external carotid artery and retromandibular vein cross the parotid parenchyma. Also, the facial nerve and its branches are found between the superficial and deep lobes of the parotid. The superficial lobe of the parotid gland cover the masseter muscle, while the submandibular gland exist in the posterior submandibular triangle which is established by anterior and posterior bellies of the digastric muscle and mandibula and it is close to the mylohyoid and hyoglossus muscles. ${ }^{[5]}$ Ultrasound guidance is superior to the blind technique, as vascular structures and muscles can be differentiated by ultrasound. However, the facial nerve cannot be clearly visible on ultrasound. ${ }^{[5]}$ Proximity to the facial nerve and its main branches can be checked by electrical stimulation. Also, to be surer that the needle tip is placed in the salivary gland parenchyma and not in muscle, electromyographic activity should be checked, as it provides auditory or visual feedback. ${ }^{[1,5]}$ If the needle tip is inside the parotid parenchyma, no activity and sound would be detected on the screen of electrical stimulation device. Therefore, electromyography \& electrical stimulation should be used rather than the blind technique with anatomical localization guidance, if the physician is not experienced in using ultrasound.

To the best of our knowledge, the injection must be done using ultrasound together with electromyography \& electrical stimulation guidance to achieve better results to increase the efficacy and decrease adverse effects such as facial asymmetry and dysphagia. Physiatrists who commonly see patients with neurological disabilities and drooling and who are already more experienced with ultrasound, electromyography \& electrical stimulation guidance than other specialties must use the three techniques together to reach better outcomes. Nonetheless, further studies evaluating the efficacy and safety of using ultrasound together with electromyography \& electrical stimulation guidance for salivary gland injections for drooling management should be conducted.

\section{Declaration of conflicting interests}

The authors declared no conflicts of interest with respect to the authorship and/or publication of this article.

\section{Funding}

The authors received no financial support for the research and/or authorship of this article.

\section{REFERENCES}

1. Lakraj AA, Moghimi N, Jabbari B. Sialorrhea: anatomy, pathophysiology and treatment with emphasis on the role of botulinum toxins. Toxins (Basel) 2013;5:1010-31.

2. Erasmus CE, van Hulst K, Rotteveel JJ, Willemsen MA, Jongerius $\mathrm{PH}$. Clinical practice: swallowing problems in cerebral palsy. Eur J Pediatr 2012;171:409-14.

3. Dogu O, Apaydin D, Sevim S, Talas DU, Aral M. Ultrasoundguided versus 'blind' intraparotid injections of botulinum toxin-A for the treatment of sialorrhoea in patients with Parkinson's disease. Clin Neurol Neurosurg 2004;106:93-6.

4. So JI, Song DH, Park JH, Choi E, Yoon JY, Yoo Y, et al. Accuracy of ultrasound-guided and non-ultrasoundguided botulinum toxin injection into cadaver salivary glands. Ann Rehabil Med 2017;41:51-7.

5. Bialek EJ, Jakubowski W, Zajkowski P, Szopinski KT, Osmolski A. US of the major salivary glands: anatomy and spatial relationships, pathologic conditions, and pitfalls. Radiographics 2006;26:745-63. 